\title{
Poor prognosis in Epstein-Barr virus-negative gastric cancer with lymphoid stroma is associated with immune phenotype
}

\author{
Charles J. Cho ${ }^{1} \cdot$ Hyo Jeong Kang ${ }^{2} \cdot$ Yeon-Mi Ryu ${ }^{3}$. Young Soo Park ${ }^{2} \cdot$ Hui Jeong Jeong ${ }^{2} \cdot$ Young-Mi Park $^{3}$. \\ Hyun Lim ${ }^{1} \cdot$ Jeong Hoon Lee ${ }^{1} \cdot$ Ho June Song ${ }^{1} \cdot$ Hwoon-Yong Jung ${ }^{1} \cdot$ Sang-Yeob Kim ${ }^{3,4} \cdot$ Seung-Jae Myung ${ }^{5}$
}

Received: 29 January 2018 / Accepted: 11 March 2018 / Published online: 7 April 2018

(c) The International Gastric Cancer Association and The Japanese Gastric Cancer Association 2018

\begin{abstract}
Background Gastric cancer with lymphoid stroma (GCLS) is pathologically characterized by poorly developed tubular structures with a prominent lymphocytic infiltration. Its clinical and prognostic features differ in patients positive and negative for Epstein-Barr virus (EBV) infection. This study analyzed the expression of programmed cell death-1 (PD-1), programmed cell death ligand-1 (PD-L1), and the density of tumor-infiltrating lymphocytes (TILs) including CD3+ and CD8+ T cells, as well as their prognostic significance in patients with GCLS.

Methods The study included 58 patients with GCLS (29 EBV+ and $29 \mathrm{EBV}-$ ) who underwent curative resection. Expression of CD3, CD8, PD-1, and PD-L1 in tumor cells and TILs was analyzed using a quantitative multispectral imaging system $\left(\mathrm{Opal}{ }^{\mathrm{TM}}\right)$, with these results validated by immuno-histochemical assays for PD-L1 on whole slide sections.

Results The proportion of tumors overexpressing PD-L1 (31.0 vs. $0 \%, P=0.002)$, TIL density (4548 vs. $2631 / \mathrm{mm}^{2}$, $P<0.001)$, and intra-tumoral CD8+ T-cell density (2650 vs. $\left.1060 / \mathrm{mm}^{2}, P<0.001\right)$ were significantly higher in EBV+ than in EBV- GCLS. In addition, CD8+/CD3+ T-cell ratio was higher in EBV+ than in EBV- GCLS (55.3 vs. 35.8\%, $P<0.001)$. Lower TIL density, defined as $<1350 / \mathrm{mm}^{2}$, was a significant negative factor of survival.

Conclusions Despite histopathological similarity, quantitative multispectral imaging revealed differences in the tumor immune micro-environment between EBV+ and EBV- GCLS, indicating that the underlying pathogenesis differs in these two disease entities. TIL density may be a prognostic marker in patients with GCLS.
\end{abstract}

Keywords Gastric cancer with lymphoid stroma $\cdot$ Immune checkpoint $\cdot$ Tumor-infiltrating lymphocyte $\cdot$ Epstein-Barr virus

Electronic supplementary material The online version of this article (https://doi.org/10.1007/s10120-018-0820-3) contains supplementary material, which is available to authorized users.

Charles J. Cho, Hyo Jeong Kang and Yeon-Mi Ryu contributed equally to this work.

Sang-Yeob Kim

sykim3yk@amc.seoul.kr

Seung-Jae Myung

sjmyung@amc.seoul.kr

1 Department of Gastroenterology, University of Ulsan College of Medicine, Asan Medical Center, Seoul 05505, Korea

2 Department of Pathology, University of Ulsan College of Medicine, Asan Medical Center, Seoul 05505, Korea

\section{Introduction}

Gastric cancer with lymphoid stroma (GCLS) is a rare histologic subtype of gastric cancer distinguished by densely packed tumor cells with massive infiltration of lymphocytes into the tumor and surrounding stroma [1-3]. GCLS can be divided into two subtypes, consisting of tumors positive and negative for Epstein-Barr virus (EBV) infection, which are

3 Asan Institute for Life Sciences, University of Ulsan College of Medicine, Asan Medical Center, Seoul 05505, Korea

4 Department of Convergence Medicine, University of Ulsan College of Medicine, Asan Medical Center, Seoul 05505, Korea

5 Department of Gastroenterology and Convergence Medicine, University of Ulsan College of Medicine, Asan Medical Center, Seoul 05505, Korea 
histologically indistinguishable. Most GCLSs are positive for EBV infection (EBV+), as shown by EBV in situ hybridization (ISH). EBV+ GCLSs are predominantly located in the upper third of the stomach, show earlier onset and better prognosis than conventional gastric adenocarcinomas $[4,5]$. In contrast, the clinical characteristics and prognosis of EBV- GCLS are comparable to those of conventional adenocarcinoma of the stomach [5]. Despite greater understanding of the clinicopathological characteristics of this disease, the mechanisms and/or factors underlying their carcinogenesis have not been determined.

Higher number of tumor-infiltrating lymphocytes (TILs) have been associated with better prognosis in several types of cancer [6-9]. Tumors have, therefore, been classified by micro-environment-related parameters, such as Immunoscore, and the treatment of several types of cancer by adoptive T-cell transfer has been attempted [10-13]. Also, EBV-associated gastric cancer (EBVaGC) frequently shows programmed cell death ligand-1 (PD-L1) overexpression and amplification of chromosome 9p, which contains the CD274, PDCD1LG2, and JAK2 genes [14-16].

Considering that lymphocytes are heavily infiltrated in GCLS and that EBV+ tumors comprise the majority of GCLS, we reasoned that the distribution of immune cells (CD8+ and/or CD3+ T cells) and the immune checkpoint modulators such as programmed cell death-1 (PD-1) and PD-L1 may affect the clinical characteristics and prognosis of patients with GCLS. This study, therefore, analyzed PD-1 and PD-L1 expression, as well as intra-tumoral CD8+ and/ or CD3+ T-cell density, in GCLS using quantitative multispectral imaging (MSI), which enables simultaneous examination of six distinct markers. Further, this study assessed whether these factors have prognostic impact in patients with GCLS.

\section{Methods}

\section{Study patients}

This study retrospectively analyzed hematoxylin and eosin (H\&E) stained slides of paraffin-embedded samples from 289 patients with GCLS who underwent gastric resection with curative intent in our center between 1998 and 2012, as previously described [5]. Only the 215 patients with GCLS component $\geq 70 \%$ were included in this study, 186 patients with EBV+ GCLS and 29 patients with EBV- GCLS. Twenty-nine EBV+ GCLS patients were randomly selected by simple random sampling using SAS software, version 9.1 (SAS Institute, Cary, NC, USA) for comparison with the 29 patients with EBV- GCLS (Supplementary Fig. 1). After random selection, baseline characteristics and prognoses of the selected EBV+GCLS patients $(n=29)$ were compared with those of non-selected EBV+ GCLS patients $(n=157)$. There was no significant difference in the baseline characteristics between selected and non-selected cases (Supplementary Table 1). Also, the 5-year survival rate (YSR) of selected patients did not significantly differ from 5-YSR of non-selected patients (96.4 vs. $96.1 \%, P=0.608$ ). The study protocol was approved by the Institutional Review Board of Asan Medical Center. (IRB No. 2017-0133).

\section{Pathologic analysis}

All cases of H\&E slides were examined by two experienced gastrointestinal pathologists (YSP, HJK). For each patient, a single key slide was selected with the following criteria: (1) the highest GCLS proportion with the deepest tumor invasion, (2) well-defined tumor center and invasive margin, (3) well-defined tumor invasive margin and non-neoplastic tissue, and (4) no necrosis or hemorrhage (Fig. 1a, upper). Identical whole-slide sections were used for immunohistochemical (IHC) staining, Opal ${ }^{\mathrm{TM}}$ staining, and EBV ISH.

\section{EBV ISH}

EBV ISH was performed using the Bench Mark XT autostainer (Ventana Medical Systems, Tucson, AZ, USA) and Ventana ISH iVIEW Blue Detection Kit (Ventana Medical Systems), according to the manufacturer's instructions. Briefly, 4- $\mu \mathrm{m}$-thick whole-slide sections, obtained with a microtome, were transferred onto silanized slides and allowed to dry for $10 \mathrm{~min}$ at room temperature, followed by $20 \mathrm{~min}$ in an incubator at $65{ }^{\circ} \mathrm{C}$. The sections were treated with protease $\mathrm{Z}$ for $8 \mathrm{~min}$, and incubated for $2 \mathrm{~h}$ with EBER probe (800-2842, Ventana Medical Systems) in the autoimmunostainer. Dark blue/purple at the site of hybridization (nucleus) was interpreted as positive for EBV (Fig. 1a, lower).

\section{IHC staining and evaluation}

Fifty-eight cases of key formalin-fixed paraffin-embedded (FFPE) blocks were immunohistochemically stained with antibody to PD-L1 (rabbit monoclonal E1L3 N, 1/100; Cell Signaling, Danvers, MA, USA) using the Bench Mark XT automatic immunostaining device with an OptiView DAB IHC Detection Kit (Ventana Medical Systems) according to the manufacturer's instructions. Serial $4-\mu$ m-thick sections were transferred onto silanized slides and allowed to dry for $10 \mathrm{~min}$ at room temperature, followed by $20 \mathrm{~min}$ in an incubator at $65^{\circ} \mathrm{C}$, subjected to heat-induced epitope retrieval using Cell Conditioning 1 (CC1) buffer for $32 \mathrm{~min}$, and incubated for 16 min with anti-PD-L1 antibody in the autoimmunostainer. Results of immunostaining were independently 
a
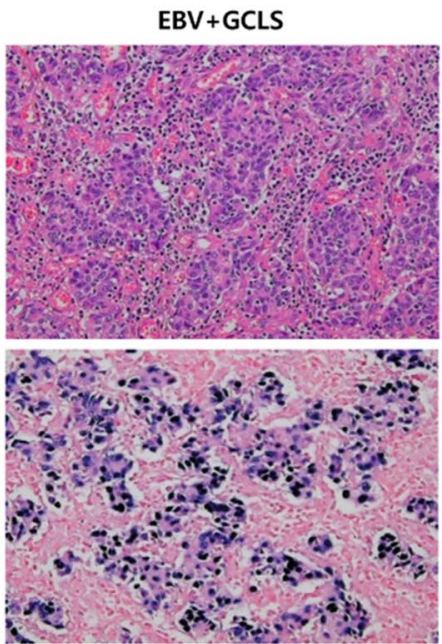

d

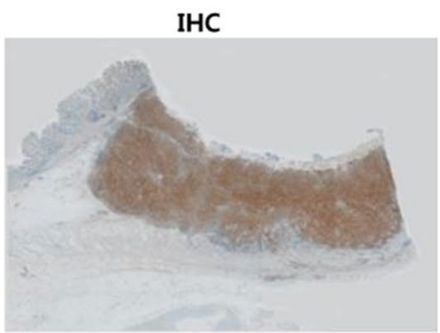

e

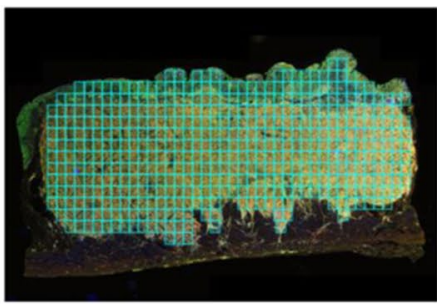

EBV-GCLS
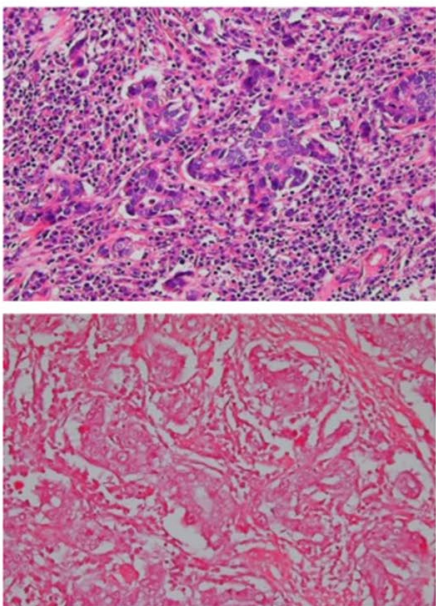

OPAL
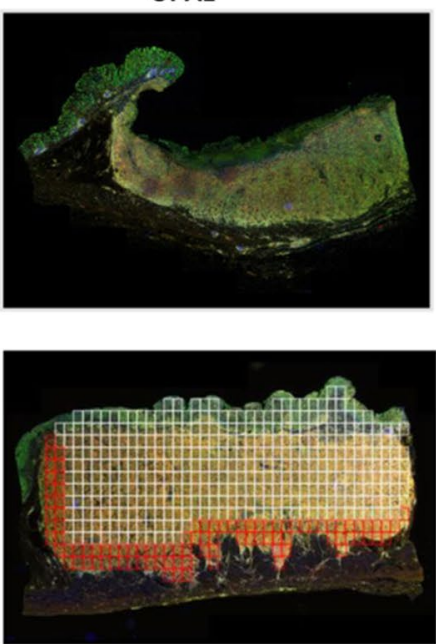

b

CK

CD8

PD-L1
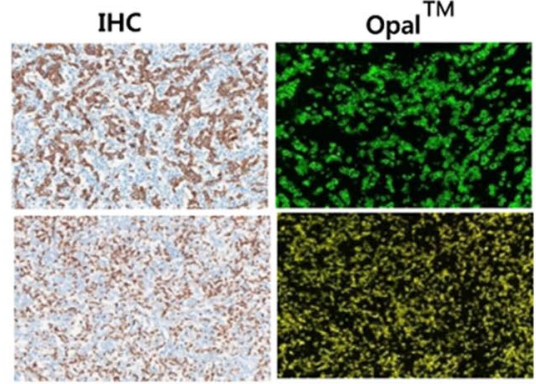

PD-1
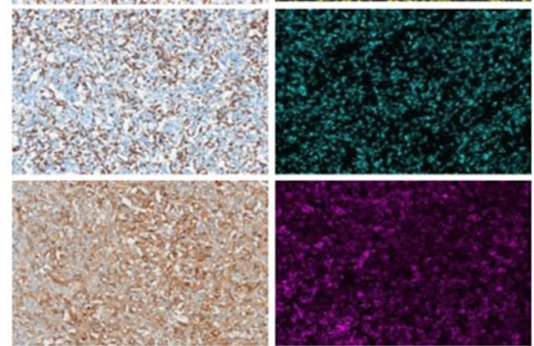

C

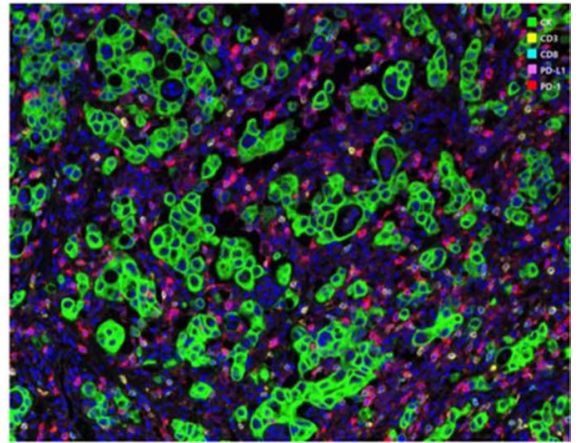

Fig. 1 Images of histologic features, IHC and Opal ${ }^{\mathrm{TM}}$ stainings in EBV+ and EBV- GCLS. a Histologically indistinguishable EBV+ and EBV- GCLS subtypes (upper) show opposite EBV ISH results. (lower) b The results of Opal ${ }^{\mathrm{TM}}$ staining for CK, CD3, CD8, PD-L1, and PD-1 well-reflect the results of corresponding IHC staining images. c Multiplex staining in GCLS tissue sections; each com-

evaluated by two pathologists (HJK, HJJ), who were blinded to clinical outcomes. PD-L1 expression was assessed in tumor cells and intra-tumoral lymphocytes. PD-L1 expression in tumor and immune cells was scored by the Immunoreactivity Scoring System (IRS), based on the percentage of stained cells and staining intensity (Supplementary Table 2) [16]. PD-L1 expression in immune cells was categorized by the percentage of $\mathrm{PD}-\mathrm{L} 1$ positive cells.

\section{Multiplex immunofluorescence staining}

Four-micrometer-thick whole-slide sections, obtained with a microtome, were transferred onto plus charged slides, followed by multiplex immunofluorescence staining with ponent distinctively expresses its unique wavelength in the merged image. d Similar pattern of IHC and Opal ${ }^{\mathrm{TM}}$ staining for PD-L1 performed in the whole tumor sections. e The analyses were performed in whole tumor area before (left, sky blue) and after dichotomizing whole tumor into the tumor center (white) and invasive margin (red) in the scanned image of the Opal ${ }^{\mathrm{TM}}$ stained slide (right)

a Leica Bond $\mathrm{Rx}^{\mathrm{TM}}$ Automated Stainer (Leica Biosystems, Newcastle, UK). Briefly, the slides were baked for 30 min and dewaxed with Leica Bond Dewax solution (Cat \#AR9222, Leica Biosystems, Milton Keynes, UK), followed by antigen retrieval with Bond Epitope Retrieval 2 (Cat \#AR9640, Leica Biosystems, Milton Keynes, UK) in a $\mathrm{pH} 9.0$ solution for $30 \mathrm{~min}$.

Multiplex immunofluorescence staining was performed using the Opal ${ }^{\mathrm{TM}}$ 7-color Automation IHC Kit (Perkin Elmer, Waltham, MA, USA). PD-1 (ab137132, AbCam, Cambridge, MA, USA) expression was visualized by Opal ${ }^{\mathrm{TM}} 690$ tyramide signal amplification (TSA) Plus (dilution, 1:300); PD-L1 (13684, CST, Danvers, MA, USA) by Opal ${ }^{\text {TM }} 650$ TSA Plus (dilution, 1:150); 
CD8 (MCA1817, AbD Serotec, Oxford, UK) by Opal ${ }^{\mathrm{TM}}$ 570 TSA Plus (dilution, 1:300); CD3 (Ventana, Tucson, AZ, USA) by Opal ${ }^{\mathrm{TM}} 650$ TSA Plus (dilution, 1:150); and cytokeratin (nbp-2-29429, Novus Biologicals, Cambridge, UK) by Opal ${ }^{\text {TM }} 520$ TSA Plus (dilution, 1:100).

Each section was subjected to five sequential rounds of staining, each including a protein block with PKI blocking/antibody diluent, followed by incubation with primary antibody and corresponding secondary horseradish peroxidase-conjugated polymer using Opal ${ }^{\mathrm{TM}}$ Polymer HRP Ms + Rb kit (Perkin Elmer, Waltham, MA, USA). Each horseradish peroxidase-conjugated polymer mediated the covalent binding of a different fluorophore using TSA. This covalent reaction was followed by additional antigen retrieval with Bond Epitope Retrieval 1 (Cat \#AR9961, Leica Biosystems, Milton Keynes, UK) for $20 \mathrm{~min}$ to remove bound antibodies before the next step in the sequence. After five sequential reactions, sections were counterstained with DAPI and coverslipped using HIGHDEF ${ }^{\circledR}$ IHC fluoromount (Enzo Life Sciences, Farmingdale, NY, USA).

\section{Multispectral imaging}

Multiplex stained slides were scanned using the Vectra ${ }^{\circledR}$ Multispectral Imaging System version 3 (Perkin Elmer, Boston, MA, USA). Each $200 \mathrm{X}$ multispectral image cube was created by combining images obtained at $10 \mathrm{~nm}$ intervals of the emission light spectrum across the range of each emission filter cube. Filter cubes used for MSI were DAPI (440-680 nm), FITC (520-680 nm), Cy3 (570-690 nm), Texas Red (580-700 nm), and Cy5 (670-720 nm).

\section{Spectral unmixing}

A spectral library containing the emitting spectral peaks of all fluorophores was created using inForm 2.2.1 image analysis software (Perkin Elmer, Wellesley, MA, USA), based on multispectral images obtained from single stained slides for each marker and associated fluorophore (Fig. 1b). This spectral library was used to separate each multispectral image cube into its individual components (spectral unmixing), allowing for the color-based identification of all six markers of interest in a single image using inForm 2.2.1 image analysis software (Fig. 1c). Quantitative measurement of the area occupied by the inflammatory cells in the scanned image was also performed with the inForm 2.2.1 software. All spectrally unmixed and segmented images were analyzed using inForm and Spotfire ${ }^{\mathrm{TM}}$ software (TIBCO Software Inc., Palo Alto, CA, USA).

\section{Analysis with Spotfire ${ }^{\mathrm{TM}}$ software and compartmentalization of the samples}

The data obtained from inForm were sent to Spotfire ${ }^{\mathrm{TM}}$ software and threshold for positivity of each factor was determined based on IHC scoring methods (Fig. 1d). Scores indicating positivity were $>2.8$ for $\mathrm{CK},>4.6$ for $\mathrm{CD} 3,>6.0$ for $\mathrm{CD} 8,>1.5$ for PD-1, and $>10.0$ for PD-L1. All cells in each slide were designated as positive or negative for each antibody, and the data were categorized and exported to an xls file for analysis. For the analysis of percentage or density of cells, whole tumor area was divided into multiple fields, which is defined as an equal rectangular unit composed of 1,349,376 pixels (Fig. 1e, left). Also, whole tumor was dichotomized into the tumor center and invasive margins and were evaluated for each component (Fig. 1e, right). A mean of $129.7 \pm 86.8$ fields was analyzed in total tumor area, $119.4 \pm 64.6$ from EBV- GCLS tumors and 139.9 \pm 104.6 from EBV+ GCLS tumors $(P=0.373)$. Tumor centers were analyzed in mean $113.1 \pm 83.0$ fields, $101.8 \pm 60.6$ from EBV - GCLS and $124.5 \pm 100.5$ from EBV+ GCLS tumors $(P=0.303)$, and invasive margins in mean $16.5 \pm 7.3$ fields, $17.6 \pm 7.5$ from EBV- GCLS and $15.4 \pm 7.0$ from EBV+ GCLS tumors $(P=0.261)$. The formula used to convert cell density from cell number per field to cell number per $\mathrm{mm}^{2}$ is as follows: cell number per $\mathrm{mm}^{2}=$ cell number per field $/ 0.36$.

\section{Statistical analysis}

Continuous variables and proportions were compared using the independent T, Mann-Whitney $U$, Chi-square, or Fisher's exact test, as appropriate. To assess the association between data, Pearson's correlation coefficient was used. Receive operating characteristic (ROC) curve analysis was used to determine the appropriate cutoff of TIL density affecting survival. The highest Youden index (sensitivity + specificity -1 ) was calculated to determine the best cutoff value. The probability of overall survival (OS) and disease-free survival (DFS) was estimated using the Kaplan-Meier method and compared using Cox proportional hazards regression models. A backward elimination approach involving candidate variables with $P \leq 0.10$ on univariate analysis was used for multivariable analysis. Two-tailed $P$ values $<0.05$ were considered statistically significant. All statistical analyses were performed using SPSS 22.0 software (SPSS Inc., Chicago, IL, USA).

\section{Results}

\section{Clinicopathologic characteristics of the patients}

The baseline demographic and clinical characteristics of the selected patients are shown in Table 1. Patients with 
EBV- GCLS were older, and lesions were more frequently located in the lower third of the stomach. Gender, stage, and lymphovascular and perineural invasion status did not differ between the two groups. Patients with EBV- GCLS showed a significantly lower 5-YSR than patients with EBV+ GCLS (79.4 vs. 96.4\%, $P=0.032$, Supplementary Fig. 2). Subgroup analysis with stage II or III GCLS patients also showed worse prognosis in EBV- GCLS (5-YSR 63.9 vs. $94.4 \%, P=0.030)$. DFS between EBV - GCLS and EBV+ GCLS were not statistically significant (5-YSR 79.4 vs. $96.4 \%, P=0.059$ ).

We then analyzed the baseline characteristics and prognoses of whole GCLS population ( $n=215$; 29 EBV- GCLS and $186 \mathrm{EBV}+\mathrm{GCLS})$ to determine whether the selected patients could be representative for whole GCLS population. Indeed, the baseline characteristics of the whole GCLS population were well-reflected by selected patients (Supplementary Table 3). 5-YSR of EBV- GCLS group was also lower than EBV+ GCLS group in whole GCLS population (79.4 vs. $96.2 \%, P=0.003$ ).

\section{Expression of PD-L1 in EBV+ GCLS and EBV- GCLS}

When PD-L1 expressing tumor cell percentage was compared, EBV+ GCLS showed a trend toward higher PD-L1 positive tumor cell percentage than EBV- GCLS [6.0\% (IQR, 1.5-31.4) vs. 3.3\% (IQR, 0.9-12.0), $P=0.179]$.
Further, PD-L1 overexpression, defined as $\geq 25 \%$ PD-L1 positive cells, was significantly more frequent in $\mathrm{EBV}+\mathrm{GCLS}$ than in EBV- GCLS (31.0 vs. $0 \%, P=0.002$; Fig. 2a). Dichotomization into tumor centers and invasive margins showed that PD-L1 overexpression was significantly more frequent in the center of EBV+ GCLS than of EBV- GCLS (27.6 vs. $0 \%, P=0.004$; Fig. 2 b), but that the difference was not significant at the invasive margins (17.2 vs. 10.3\%, $P=0.706$; Fig. 2c). Similar findings were observed by IHC, with higher IRS score in EBV+ GCLS than in EBV - GCLS $(3.2 \pm 2.0$ vs. $1.9 \pm 1.7, P=0.007)$. In addition, PD-L1 overexpression, defined as $\mathrm{IRS} \geq 6$, being significantly more frequent in EBV+ GCLS than in EBV- GCLS (20.7 vs. $0 \%, P=0.023$; Fig. 2d). Analysis of concordance between the two methods for detection of PD-L1 overexpression showed a good strength of agreement (kappa agreement coefficient of 0.772). We also investigated whether lymphocyte PD-L1 positivity differed in EBV+ GCLS and EBV - GCLS. Intra-tumoral PD-L1+ lymphocytes, as determined by $\operatorname{IRS}$ score $\geq 2$, were significantly more frequent in EBV+ GCLS than in EBV- GCLS (51.7 vs. $17.2 \%, P=0.006$; Fig. 2 e).
Table 1 Comparison of clinicopathological characteristics between $\mathrm{EBV}+\mathrm{GCLS}$ and EBV- GCLS

\begin{tabular}{|c|c|c|c|}
\hline Variables & $\begin{array}{l}\text { EBV-GCLS }(\%) \\
(n=29)\end{array}$ & $\begin{array}{l}\text { EBV+GCLS }(\%) \\
(n=29)\end{array}$ & $P$ value \\
\hline Age, year, mean \pm SD & $61.2 \pm 10.4$ & $54.4 \pm 12.8$ & 0.03 \\
\hline Sex & & & 0.517 \\
\hline Male & $22(75.9)$ & $24(82.8)$ & \\
\hline Female & $7(24.1)$ & $5(17.2)$ & \\
\hline Tumor location & & & 0.001 \\
\hline Upper & $3(10.3)$ & $5(17.2)$ & \\
\hline Middle & $10(34.5)$ & $21(72.4)$ & \\
\hline Lower & $16(55.2)$ & $3(10.3)$ & \\
\hline TNM stage & & & 0.935 \\
\hline Stage I & $12(41.4)$ & $11(37.9)$ & \\
\hline Stage II & $12(41.4)$ & $12(41.4)$ & \\
\hline Stage III-IV & $5(17.2)$ & $6(20.7)$ & \\
\hline Lymphovascular invasion & & & 0.773 \\
\hline Present & $9(31.0)$ & $8(27.6)$ & \\
\hline Absent & $20(69.0)$ & $21(72.4)$ & \\
\hline Perineural invasion & & & 0.738 \\
\hline Present & $6(20.7)$ & $5(17.2)$ & \\
\hline Absent & $23(79.3)$ & $24(82.8)$ & \\
\hline Adjuvant treatment & & & 0.594 \\
\hline Yes & $11(37.9)$ & $13(44.8)$ & \\
\hline No & $18(62.1)$ & $16(55.2)$ & \\
\hline
\end{tabular}



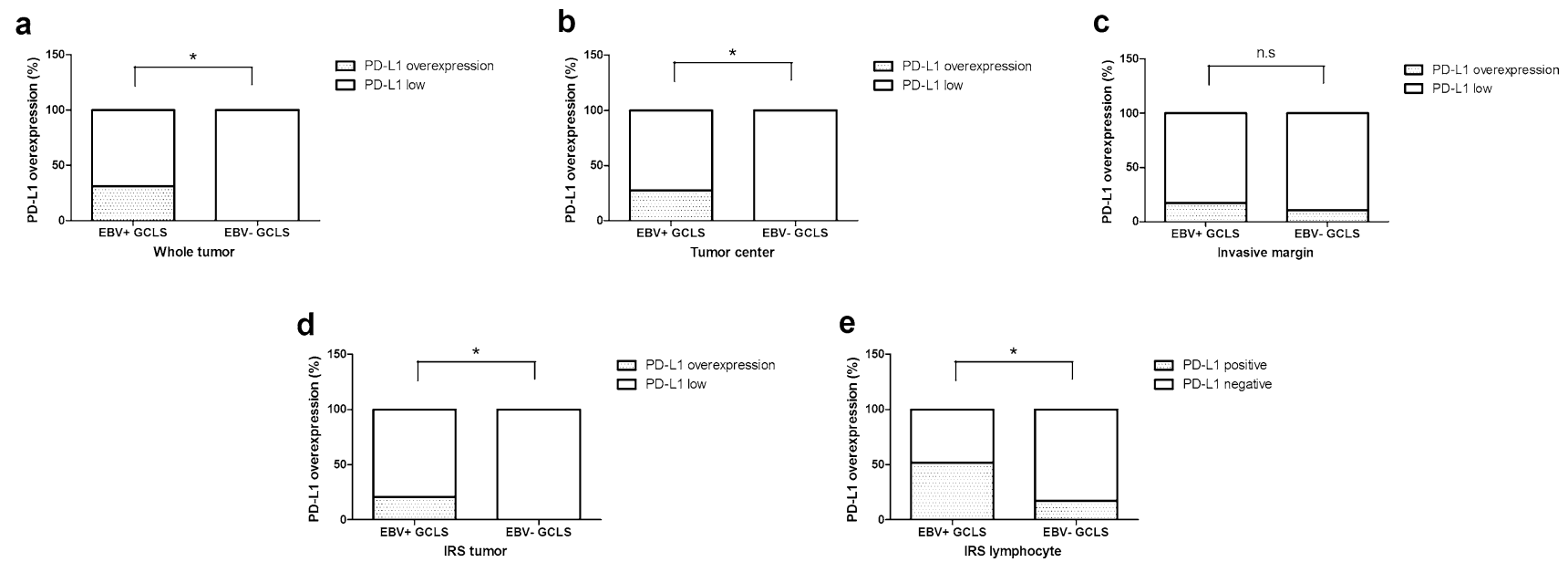

Fig. 2 Frequency of PD-L1 overexpression in EBV+GCLS and EBV- GCLS. PD-L1 overexpression as determined by a quantitative multispectral imaging system $\left(\mathrm{Opal}^{\mathrm{TM}}\right)$ in $\mathbf{a}$ whole tumor sections, $\mathbf{b}$

\section{TIL quantity and infiltration pattern in EBV+ GCLS and EBV-GCLS}

TILs have been associated with favorable clinical outcomes and clinical response in various solid tumors. We, therefore, assessed the density of TILs using CD3+T cell counts in GCLS. CD8+ T cell, which is known to play a central role in adaptive immune resistance associated with PD-L1 upregulation via type II interferon secretion, were also quantified. We found that CD3+ T-cell density was significantly higher in whole tumors $(4547.6 \pm 1997.9$ vs. $2631.3 \pm 1879.2 / \mathrm{mm}^{2}, P<0.001$; Fig. 3a), tumor centers $\left(4478.8 \pm 1941.1\right.$ vs. $2586.6 \pm 1911.2 / \mathrm{mm}^{2}, P<0.001$; Fig. $3 \mathrm{~b})$, and invasive margins (5050.5 \pm 2655.8 vs. $3126.4 \pm 2963.6 / \mathrm{mm}^{2}, P=0.012$; Fig. 3c) of EBV+ GCLS than of EBV- GCLS samples. Similar results were observed for CD8+ T-cell density, which was significantly higher in whole tumors $\left(2650.1 \pm 1621.0\right.$ vs. $1060.0 \pm 1066.6 / \mathrm{mm}^{2}$, $P<0.001$; Fig. 3d), tumor centers $(2617.8 \pm 1579.0$ vs. $1017.9 \pm 1049.2 / \mathrm{mm}^{2}, P<0.001$; Fig. 3 e), and invasive margins [2128.4 (IQR, 1319.0-4099.2) vs. 915.4 (IQR, 310.0-1592.6) $/ \mathrm{mm}^{2}, P<0.001$; Fig. 3f] of EBV+ GCLS than of EBV- GCLS samples. In addition, CD8+/CD3+T cell ratios were significantly higher in whole tumors (55.3 vs. $35.8 \%, P<0.001$; Fig. $3 \mathrm{~g}$ ), tumor centers (55.9 vs. $34.9 \%$, $P<0.001$; Fig. 3 h), and invasive margins (53.5 vs. $38.8 \%$, $P=0.002$; Fig. 3i) of EBV+ GCLS than of EBV- GCLS samples.

\section{Effects of EBV infection and compartment on PD-L1+ TIL quantity and distribution pattern}

Median PD-L1+CD3+T-cell densities were significantly higher in whole tumors [513.8 (IQR, 107.7-1163.6) vs. tumor centers, and c invasive margins. d PD-L1 overexpression as determined by IHC in whole tumor sections. e PD-L1 positivity in intra-tumoral lymphocytes assessed by IHC

143.7 (IQR, 71.4-526.3) $/ \mathrm{mm}^{2}, P=0.015$; Fig. 4a] and in tumor centers [555.0 (IQR, 126.2-1233.2) vs. 117.2 (IQR, $57.8-388.2) / \mathrm{mm}^{2}, P=0.003$; Fig. $\left.4 \mathrm{~b}\right]$ of EBV+ GCLS than of EBV- GCLS samples, a finding not observed in the invasive margins of the two groups [294.3 (IQR, 135.6-926.1) vs. 203.5 (IQR, 50.0-754.5)/mm²,$P=0.253$; Fig. 4c]. The distribution of PD-L1+CD8+ T cells showed a similar pattern. PD-L1+CD8+ T-cell densities were significantly higher in whole tumors [230.3 (IQR, 55.9-853.1) vs. 56.9 (IQR, 23.0-218.4)/mm² $P=0.004$; Fig. $4 \mathrm{~d}]$ and in tumor centers [249.0 (IQR, 55.9-899.6) vs. 45.8 (IQR, $21.3-149.6) / \mathrm{mm}^{2}, P=0.001$; Fig. 4e] of EBV+ GCLS than of EBV- GCLS samples, a finding not observed in their invasive margins [135.6 (IQR, 59.3-608.8) vs. 87.5 (IQR, 14.3-397.2) $/ \mathrm{mm}^{2}, P=0.092$; Fig. 4f].

\section{Effect of EBV infection on PD-1+CD8+ intra-tumoral T-cell density}

We further assessed whether PD-1 positive T-cell density differed in EBV+ and EBV- GCLS samples. PD-1+CD8+T-cell density was significantly higher in whole tumors [522.4 (IQR, 305.5-1068.3) vs. 287.4 (IQR, 70.1-776.6) $/ \mathrm{mm}^{2}, P=0.011$; Supplementary Fig. 3a], tumor centers [533.3 (IQR, 298.9-1014.4) vs. 249.9 (IQR, 56.6-626.1) $/ \mathrm{mm}^{2}, P=0.008$; Supplementary Fig. 3b], and invasive margins [679.2 (IQR, 355.8-1140.8) vs. 338.3 (IQR, 85.2-650.8) $/ \mathrm{mm}^{2}, P=0.019$; Supplementary Fig. 3c] of EBV+ GCLS than of EBV- GCLS samples. However, the PD-1+CD3+ T-cell density in whole tumors of EBV+ GCLS and EBV- GCLS samples did not differ significantly $\left(1277.7 \pm 1044.3\right.$ vs. $833.8 \pm 631.4 / \mathrm{mm}^{2}$, $P=0.055$; Supplementary Fig. 3d). 
a

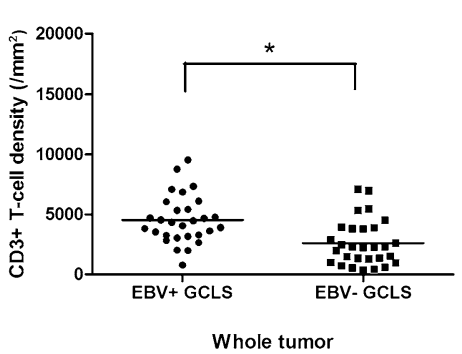

d

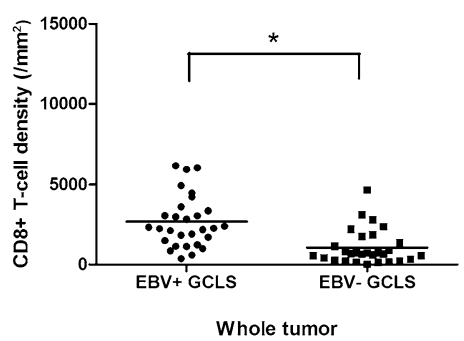

g

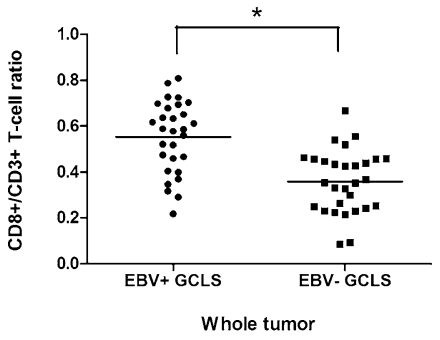

b

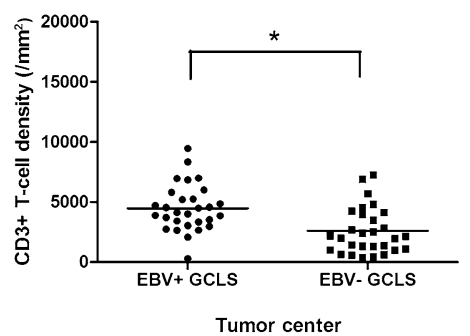

e

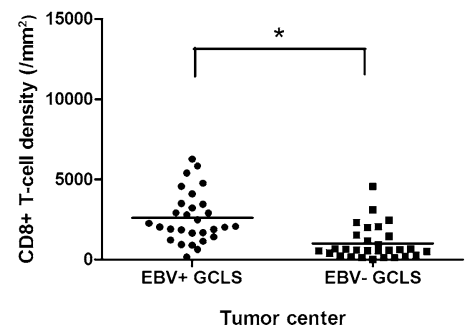

h

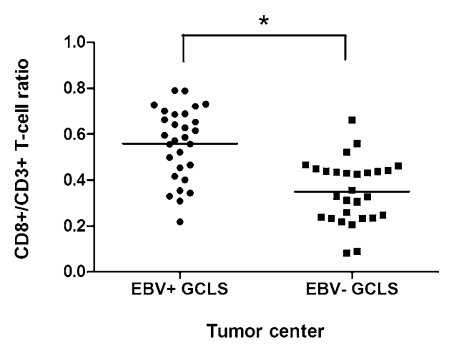

C

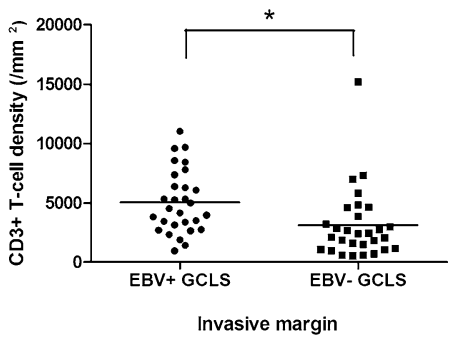

f

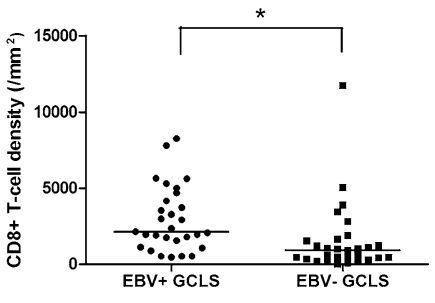

Invasive margin

i

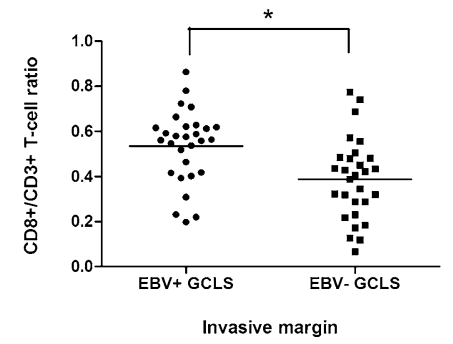

Fig. 3 TIL quantity and infiltration pattern in EBV+GCLS and EBV- GCLS. CD3+T-cell densities in a whole tumor sections, b tumor centers, and $\mathbf{c}$ invasive margins of EBV+ GCLS and EBVGCLS samples. CD8+T-cell densities in $\mathbf{d}$ whole tumor sections, e tumor centers, and $\mathbf{f}$ invasive margins of EBV+ GCLS and EBVGCLS samples. (g-i) CD3+/CD8+T-cell ratios in $\mathbf{g}$ whole tumor sections, $\mathbf{h}$ tumor centers, and $\mathbf{i}$ invasive margins of EBV+GCLS and EBV-GCLS samples

\section{Correlation between PD-L1+ tumor cell proportion and PD-L1+CD8+ T-cell density}

Correlation analyses were performed to determine the relationships of PD-L1+ tumor cell proportion with TIL density and PD-L1 status. When assessed in all patients $(n=58)$, the proportion of PD-L1+tumor cells showed significant positive correlations with PD-L1+CD3+T-cell density $(R=0.682, P<0.001$; Supplementary Fig. $4 \mathrm{a})$ and PD-L1+CD8+T-cell density $(R=0.694, P<0.001$; Supplementary Fig. $4 \mathrm{~b})$. In addition, we observed a significant correlation between the densities of PD-L1+CD8+ and PD- $1+$ CD8 + T-cells $(R=0.651, P<0.001$; Supplementary Fig. $4 c)$, with this correlation being stronger in
EBV-GCLS $(R=0.826, P<0.001)$ than in EBV+ GCLS $(R=0.576, P=0.001)$.

\section{Prognostic impact of TIL density in GCLS patient survival}

Finally, we assessed whether tumor PD-L1 overexpression or TIL density are prognostic factors in GCLS. Using the Youden index, we calculated that a cutoff of $1350 / \mathrm{mm}^{2}$ represented the point with the largest area under curve for patient survival. The 5-YSR was significantly higher in patients with a TIL density $\geq 1350 / \mathrm{mm}^{2}$ than in those with a TIL density $<1350 / \mathrm{mm}^{2}$ (92.8 vs. $64.8 \%, P=0.001$; Fig. 5 ). In contrast, 5-YSR in the two groups was independent of 
a

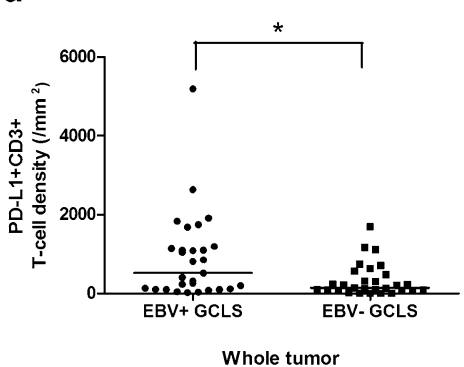

d

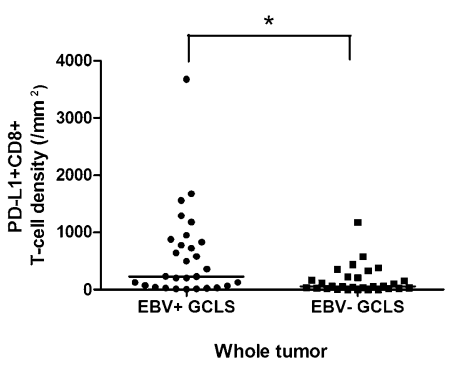

b

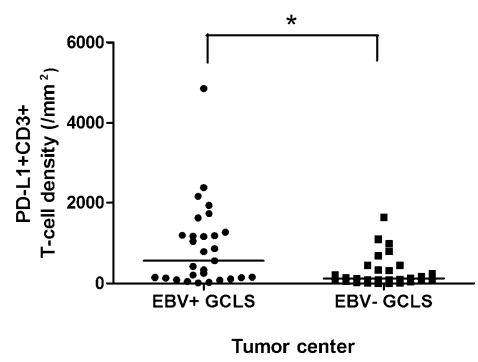

e

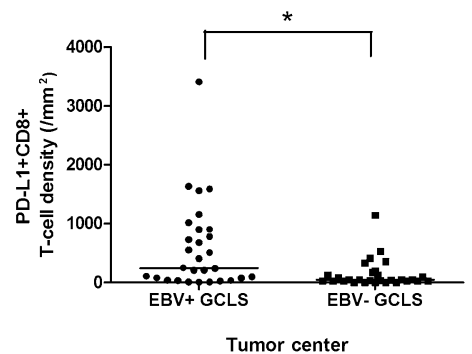

C

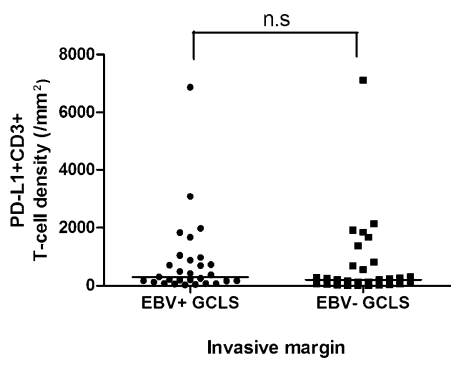

$\mathbf{f}$

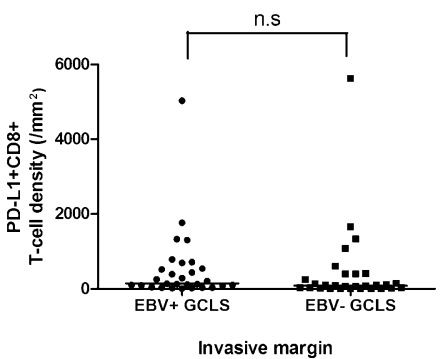

Fig. 4 Effects of EBV infection and compartment on PD-L1+TIL quantity and distribution pattern. PD-L1+CD3+T-cell densities in a whole tumor sections, $\mathbf{b}$ tumor centers, and $\mathbf{c}$ invasive margins of

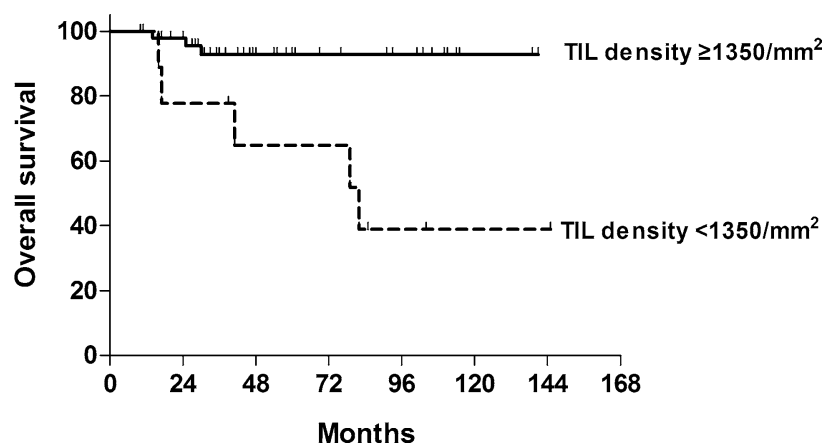

Fig. 5 Overall survival of the GCLS patients according to TIL density Kaplan-Meier analysis of overall survival in patients with TIL density $<1350 / \mathrm{mm}^{2}$ and $\geq 1350 / \mathrm{mm}^{2}$. ( $P$ values in log-rank test)

overexpression of tumor PD-L1 (87.6 vs. 87.5\%, $P=0.963)$. Univariate analysis of factors such as TIL density and tumor PD-L1 overexpression, as well as factors reported prognostic in patients with GCLS, including age, tumor stage, and presence of lymphovascular and perineural invasion, showed that advanced tumor stage and TIL density $<1350 / \mathrm{mm}^{2}$ were significant negative predictors of OS. In the multivariate analysis, tumor stage III or IV $(P=0.02)$ and TIL density $<1350 / \mathrm{mm}^{2}(P=0.04)$ remained significant negative predictors of OS (Table 2). Further, subgroup analyses were performed to assess whether TIL density is a prognostic factor in EBV - GCLS or EBV+ GCLS subgroup,
EBV+ GCLS and EBV- GCLS samples. PD-L1+CD8+ T-cell densities in $\mathbf{d}$ whole tumor sections, $\mathbf{e}$ tumor centers, and $\mathbf{f}$ invasive margins of EBV+ GCLS and EBV- GCLS samples

respectively. Among EBV - GCLS patients $(n=29)$, the 5 -YSR of patients with TIL density $<1350 / \mathrm{mm}^{2}$ was significantly lower than that of patients with TIL density $\geq 1350$ / $\mathrm{mm}^{2}$ (60.0 vs. $87.7 \%, P=0.018$ ). There was no significant difference in survival of the patients according to TIL density classification in EBV + GCLS due to small number of death cases $(P=0.847)$.

\section{Discussion}

This study showed that tumor PD-L1 overexpression and TIL infiltration profiles differ in GCLS with and without EBV infection. Intra-tumoral TIL density $<1350 / \mathrm{mm}^{2}$ was a significant negative prognostic factor, along with advanced tumor stage.

Both B- and T-lymphocytes express PD-L1 and PD-1 [17, 18]. PD-1 expression on activated T-lymphocytes may lead to PD-L1 expression on tumor cells. Interactions between tumor PD-L1 and lymphocyte PD-1 may result in the exhaustion of $T$ cells and constitute a mechanism by which tumor cells evade immune surveillance. Patients with these tumors may, therefore, benefit from a blockade of PD-1 or PD-L1 [19-22].

The effects of PD-L1 expression and TIL density on the prognosis of patients with gastric cancer have been found to differ among studies. For example, a Japanese study of 105 
Table 2 Univariate and multivariate analyses of overall survival according to clinicopathological variables

\begin{tabular}{|c|c|c|c|c|c|c|}
\hline \multirow[t]{2}{*}{ Variables } & \multicolumn{3}{|c|}{ Univariate analysis } & \multicolumn{3}{|c|}{ Multivariate analysis } \\
\hline & $P$ & $\mathrm{HR}$ & $95 \%$ CI & $P$ & HR & $95 \% \mathrm{CI}$ \\
\hline Age, +1 year & 0.15 & 1.05 & $0.98-1.13$ & - & - & - \\
\hline Tumor stage, III or IV vs. I or II & 0.003 & 9.24 & $2.17-39.38$ & 0.02 & 5.90 & $1.30-26.69$ \\
\hline Lymphovascular invasion, yes vs. no & 0.14 & 2.89 & $0.72-11.64$ & - & - & - \\
\hline Perineural invasion, yes vs. no & 0.20 & 2.58 & $0.62-10.85$ & - & - & - \\
\hline PD-L1 overexpression, yes vs. no & 0.96 & 0.95 & $0.12-7.76$ & - & - & \\
\hline TIL density $<1350$ vs. $\geq 1350 / \mathrm{mm}^{2}$ & 0.005 & 7.79 & $1.85-32.83$ & 0.04 & 4.82 & $1.07-21.73$ \\
\hline EBV status, negative vs. positive ${ }^{a}$ & 0.067 & 7.10 & $0.87-57.72$ & & & \\
\hline
\end{tabular}

$H R$ hazard ratio, $C I$ confidence interval, $E B V$ Epstein-Barr virus

${ }^{a}$ This factor was not entered in the multivariate analysis because of its high correlation to the TIL density and risk of overfitting patients with stage II and III gastric cancer who underwent curative gastrectomy showed that OS was poorer in patients positive than negative for PD-L1. Although their EBV status was not reported, 53 of these patients had undifferentiated tumors, suggesting that some may have had GCLS [23]. In contrast, a study of 451 Caucasian patients with gastric cancer showed that high PD-L1/PD-1 expression was associated with better patient outcomes [16]. However, only $20(4.4 \%)$ of these patients were positive for EBV, resulting in limited information on PD-L1 expression in patients with GCLS. A study of PD-L1 expression and CD8+ T-cell density in 34 patients with gastric cancer in the proximal stomach and gastroesophageal junction found that, although $32(94 \%)$ were negative for EBV ISH, four (12\%) tumors showed membranous PD-L1 expression [24]. In addition, increased CD8+T-cell count was associated with increased PD-L1 expression, which negatively correlated with patient survival.

The result in the current study showed that PD-L1 overexpression was more frequent in patients with EBV+GCLS than with EBV- GCLS. However, PD-L1 overexpression status was not associated with patient prognosis. This unique pattern and implication of PD-L1 expression in GCLS may be interpreted in several ways. First, the reasons for PD-L1 overexpression may vary in EBV+GCLS. Although the only GCLSs containing $\geq 25 \%$ PD-L1-positive tumor cells were $\mathrm{EBV}+$, some EBV+ GCLSs showed no evidence of PD-L1 production either by MSI and/or IHC, which led to heterogeneity in PD-L1 expression of this group. PD-L1 overexpression by some of these tumors may be due to amplification of chromosome 9p, which harbors the gene coding PD-L1 [14]. PD-L1 expression may also be induced in response to pro-inflammatory cytokines and IFN- $\gamma$ released by TILs, via activation of the JAK-STAT pathway and IRF1 [25, 26]. TILs are strongly associated with local PD-L1 expression in melanoma [27], suggesting that PD-L1 overexpression in EBV+ GCLS may be related to their higher TIL density. Further, because epithelial and cancer cells can produce type I interferons in response to various stimuli [28, 29], EBV+ GCLS tumor cells may produce interferons that activate PD-L1 in both tumor cells and lymphocytes [30]. Indeed, we observed a positive correlation between tumor PD-L1 and TIL PD-L1 expression, suggesting that interferon production in these patients affected both tumor and lymphocyte PD-L1 expression. The data in the current study may warrant further investigation focusing on the role of interferons and its downstream signaling components such as JAK/STAT, IRF9 or interferon stimulated genes as possible therapeutic targets for EBV+ GCLS [31-33].

TIL density has been associated with a favorable prognosis in patients with gastric and other types of cancer [34-36]. In the present study, TIL density clearly differed in the EBV+ GCLS and EBV- GCLS groups, with the cutoff of $1350 / \mathrm{mm}^{2}$ as TIL density affecting survival of the GCLS patients. We also tried to assess the quantity of TILs by measuring the area occupied by the inflammatory cells in the scanned image. Although the proportion of TIL area in patients with a TIL density $\geq 1350 / \mathrm{mm}^{2}$ was larger than that in patients with a TIL density $<1350 / \mathrm{mm}^{2}$, statistical significance was not reached $(62.9 \pm 15.0$ vs. $46.3 \pm 26.5 \%$, $P=0.10)$. We, therefore, suggest that TIL density measurement may be a more reliable modality in assessing the influence of TILs over eyeball measuring under microscopy or TIL area calculation.

It is unclear why relatively fewer $\mathrm{T}$ cells were recruited in EBV- GCLS, and whether the difference was due to a difference in their underlying pathogenesis. Microsatellite instability subtypes were reported to be associated with EBV- GCLS, making this association a subject of further investigation [15, 34].

The limitations of this study include its retrospective nature and the relatively small number of patients. Some of the factors regarded as insignificant in this study may show significant differences in larger patient populations. Moreover, as all of our patients were Asian, the results of the current study may be limited to Asian populations, suggesting 
a need to assess these phenotypes in other ethnicities. To our knowledge, however, this is the first study to identify an as-yet-unknown factor associated with better prognosis in patients with EBV+ GCLS than with EBV - GCLS [37]. In addition, this study maximized the use of the MSI technique, which can simultaneously analyze cells positive for multiple markers and examine whole section slides, thereby enabling an overview of the whole landscape of tumor and immune cell populations with precision. The results of the current study provide important clues in understanding and developing treatment strategies for a rare group of patients with GCLS

In conclusion, tumor PD-L1 overexpression and TIL infiltration profiles differ in GCLSs positive and negative for EBV infection. Intra-tumoral TIL density may serve as a prognostic marker in patients with GCLS.

Acknowledgements This research was supported by a grant of the Korea Health Technology R\&D Project through the Korea Health Industry Development Institute (KHIDI), funded by the Ministry of Health \& Welfare, Republic of Korea (No. HI15C3078).

\section{Compliance with ethical standards}

Conflict of interest The authors declare they have no conflict of interest.

Human rights statement All procedures followed were in accordance with the ethical standards of the responsible committee on human experimentation (institutional and national) and with the Helsinki Declaration of 1964 and later versions.

Informed consent Informed consent to be included in the study, or the equivalent, was obtained from all patients.

\section{References}

1. Horiuchi K, Mishima K, Ohsawa M, Aozasa K. Carcinoma of stomach and breast with lymphoid stroma: localisation of EpsteinBarr virus. J Clin Pathol. 1994;47:538-40.

2. Burke AP, Yen TS, Shekitka KM, Sobin LH. Lymphoepithelial carcinoma of the stomach with Epstein-Barr virus demonstrated by polymerase chain reaction. Mod Pathol. 1990;3:377-80.

3. Song HJ, Srivastava A, Lee J, Kim YS, Kim KM, Ki Kang W, et al. Host inflammatory response predicts survival of patients with Epstein-Barr virus-associated gastric carcinoma. Gastroenterology. 2010;139(84-92):e2.

4. Nakamura S, Ueki T, Yao T, Ueyama T, Tsuneyoshi M. EpsteinBarr virus in gastric carcinoma with lymphoid stroma. Special reference to its detection by the polymerase chain reaction and in situ hybridization in 99 tumors, including a morphologic analysis. Cancer. 1994;73:2239-49.

5. Lim H, Park YS, Lee JH, Son DH, Ahn JY, Choi KS, et al. Features of gastric carcinoma with lymphoid stroma associated with Epstein-Barr virus. Clin Gastroenterol Hepatol. 2015; 13(1738-44):e2.

6. Loi S, Michiels S, Salgado R, Sirtaine N, Jose V, Fumagalli D, et al. Tumor infiltrating lymphocytes are prognostic in triple negative breast cancer and predictive for trastuzumab benefit in early breast cancer: results from the FinHER trial. Ann Oncol. 2014;25:1544-50.

7. Naito Y, Saito K, Shiiba K, Ohuchi A, Saigenji K, Nagura $\mathrm{H}$, et al. CD8+ $\mathrm{T}$ cells infiltrated within cancer cell nests as a prognostic factor in human colorectal cancer. Cancer Res. 1998;58:3491-4.

8. Fujii H, Arakawa A, Utsumi D, Sumiyoshi S, Yamamoto Y, Kitoh A, et al. CD8(+) tumor-infiltrating lymphocytes at primary sites as a possible prognostic factor of cutaneous angiosarcoma. Int J Cancer. 2014;134:2393-402

9. Fukunaga A, Miyamoto M, Cho Y, Murakami S, Kawarada Y, Oshikiri T, et al. CD8+tumor-infiltrating lymphocytes together with CD4+tumor-infiltrating lymphocytes and dendritic cells improve the prognosis of patients with pancreatic adenocarcinoma. Pancreas. 2004;28:e26-31.

10. Galon J, Pages F, Marincola FM, Angell HK, Thurin M, Lugli A et al. Cancer classification using the Immunoscore: a worldwide task force. J Transl Med. 2012;10:205.

11. Andersen R, Donia M, Ellebaek E, Borch TH, Kongsted P, Iversen $\mathrm{TZ}$, et al. Long-lasting complete responses in patients with metastatic melanoma after adoptive cell therapy with tumor-infiltrating lymphocytes and an attenuated i12 regimen. Clin Cancer Res. 2016;22:3734-45.

12. Rosenberg SA, Yang JC, Sherry RM, Kammula US, Hughes MS, Phan GQ, et al. Durable complete responses in heavily pretreated patients with metastatic melanoma using T-cell transfer immunotherapy. Clin Cancer Res. 2011;17:4550-7.

13. Tran E, Turcotte S, Gros A, Robbins PF, Lu YC, Dudley ME, et al Cancer immunotherapy based on mutation-specific CD4+ T cells in a patient with epithelial cancer. Science. 2014;344:641-5.

14. Cancer Genome Atlas Research Network. Comprehensive molecular characterization of gastric adenocarcinoma. Nature. 2014;513:202-9.

15. Derks S, Liao X, Chiaravalli AM, Xu X, Camargo MC, Solcia E, et al. Abundant PD-L1 expression in Epstein-Barr Virus-infected gastric cancers. Oncotarget. 2016;7:32925-32.

16. Boger C, Behrens HM, Mathiak M, Kruger S, Kalthoff H, Rocken C. PD-L1 is an independent prognostic predictor in gastric cancer of Western patients. Oncotarget. 2016;7:24269-83.

17. Agata Y, Kawasaki A, Nishimura H, Ishida Y, Tsubata T, Yagita $\mathrm{H}$, et al. Expression of the PD-1 antigen on the surface of stimulated mouse T and B lymphocytes. Int Immunol. 1996;8:765-72.

18. Blank C, Gajewski TF, Mackensen A. Interaction of PD-L1 on tumor cells with PD-1 on tumor-specific T cells as a mechanism of immune evasion: implications for tumor immunotherapy. Cancer Immunol Immunother. 2005;54:307-14.

19. Dong H, Strome SE, Salomao DR, Tamura H, Hirano F, Flies DB, et al. Tumor-associated B7-H1 promotes T-cell apoptosis: a potential mechanism of immune evasion. Nat Med. 2002;8:793-800.

20. Topalian SL, Hodi FS, Brahmer JR, Gettinger SN, Smith DC, McDermott DF, et al. Safety, activity, and immune correlates of anti-PD-1 antibody in cancer. N Engl J Med. 2012;366:2443-54.

21. Brahmer JR, Tykodi SS, Chow LQ, Hwu WJ, Topalian SL, Hwu $\mathrm{P}$, et al. Safety and activity of anti-PD-L1 antibody in patients with advanced cancer. N Engl J Med. 2012;366:2455-65.

22. Butte MJ, Keir ME, Phamduy TB, Sharpe AH, Freeman GJ. Programmed death-1 ligand 1 interacts specifically with the B7-1 costimulatory molecule to inhibit $\mathrm{T}$ cell responses. Immunity. 2007;27:111-22.

23. Eto S, Yoshikawa K, Nishi M, Higashijima J, Tokunaga T, Nakao $\mathrm{T}$, et al. Programmed cell death protein 1 expression is an independent prognostic factor in gastric cancer after curative resection. Gastric Cancer. 2016;19:466-71.

24. Thompson ED, Zahurak M, Murphy A, Cornish T, Cuka N, Abdelfatah E, et al. Patterns of PD-L1 expression and CD8 T cell 
infiltration in gastric adenocarcinomas and associated immune stroma. Gut. 2017;66:794-801.

25. Pardoll DM. The blockade of immune checkpoints in cancer immunotherapy. Nat Rev Cancer. 2012;12:252-64.

26. Lee SJ, Jang BC, Lee SW, Yang YI, Suh SI, Park YM, et al. Interferon regulatory factor- 1 is prerequisite to the constitutive expression and IFN-gamma-induced upregulation of B7-H1 (CD274). FEBS Lett. 2006;580:755-62.

27. Taube JM, Anders RA, Young GD, Xu H, Sharma R, McMiller $\mathrm{TL}$, et al. Colocalization of inflammatory response with B7-h1 expression in human melanocytic lesions supports an adaptive resistance mechanism of immune escape. Sci Transl Med. 2012;4:127ra37.

28. Li H, Zhang J, Kumar A, Zheng M, Atherton SS, Yu FS. Herpes simplex virus 1 infection induces the expression of proinflammatory cytokines, interferons and TLR7 in human corneal epithelial cells. Immunology. 2006;117:167-76.

29. Li K, Chen Z, Kato N, Gale M Jr, Lemon SM. Distinct poly(I-C) and virus-activated signaling pathways leading to interferon-beta production in hepatocytes. J Biol Chem. 2005;280:16739-47.

30. Muhlbauer M, Fleck M, Schutz C, Weiss T, Froh M, Blank C, et al. PD-L1 is induced in hepatocytes by viral infection and by interferon-alpha and -gamma and mediates $\mathrm{T}$ cell apoptosis. $\mathrm{J}$ Hepatol. 2006;45:520-8.

31. Muller M, Briscoe J, Laxton C, Guschin D, Ziemiecki A, Silvennoinen $\mathrm{O}$, et al. The protein tyrosine kinase JAK1 complements defects in interferon-alpha/beta and -gamma signal transduction. Nature. 1993;366:129-35.

32. Qureshi SA, Salditt-Georgieff M, Darnell JE Jr. Tyrosine-phosphorylated Stat1 and Stat 2 plus a $48-k D a$ protein all contact DNA in forming interferon-stimulated-gene factor 3. Proc Natl Acad Sci USA. 1995;92:3829-33.

33. Levy DE, Kessler DS, Pine R, Darnell JE Jr. Cytoplasmic activation of ISGF3, the positive regulator of interferon-alphastimulated transcription, reconstituted in vitro. Genes Dev. 1989;3:1362-71.

34. Grogg KL, Lohse CM, Pankratz VS, Halling KC, Smyrk TC. Lymphocyte-rich gastric cancer: associations with Epstein-Barr virus, microsatellite instability, histology, and survival. Mod Pathol. 2003;16:641-51.

35. Ishigami $S$, Natsugoe $S$, Tokuda $K$, Nakajo A, Che X, Iwashige $H$, et al. Prognostic value of intratumoral natural killer cells in gastric carcinoma. Cancer. 2000;88:577-83.

36. Loi S, Sirtaine N, Piette F, Salgado R, Viale G, Van Eenoo F, et al. Prognostic and predictive value of tumor-infiltrating lymphocytes in a phase III randomized adjuvant breast cancer trial in node-positive breast cancer comparing the addition of docetaxel to doxorubicin with doxorubicin-based chemotherapy: BIG 02-98. J Clin Oncol. 2013;31:860-7.

37. Chang MS, Kim WH, Kim CW, Kim YI. Epstein-Barr virus in gastric carcinomas with lymphoid stroma. Histopathology. 2000;37:309-15. 